Revista Facultad 2019

de Ciencias Vol. 27(1)

Económicas enero-junio 2019

ISSN: 0121-6805 · ISSN-e: 1909-7719

- pp. 185-202

DOI: https://doi.org/10.18359/rfce.3233

\title{
Los negocios internacionales y el relacionamiento económico: una revisión de la literatura*
}

\author{
Pedro Nel Valbuena Hernández ${ }^{a}$ Yamile Andrea Montenegro Jaramillob
}

\begin{abstract}
Resumen: Este artículo presenta tendencias generales de investigación de relacionamiento económico y negocios internacionales, a partir de la revisión de la literatura científica. En consecuencia, el objetivo consistió en examinar las trayectorias temáticas a partir de las publicaciones científicas que circulan en Scopus. Como método se utilizaron técnicas de minería de datos y análisis de contenido, para identificar clúster en el campo de estudio, en una ventana de observación que abarca cerca de tres décadas de publicaciones, identificando tópicos centrales y jerarquizados. El alcance de este artículo está orientado a mostrar una visión general del estado actual de producción de conocimiento que permita a los académicos e investigadores orientar futuras investigaciones en el campo de los negocios internacionales. Los seis grupos más importantes fueron: primero, procesos de expansión y estudios sobre la empresa; segundo, productividad y comportamiento de exportación; tercero, sobre relaciones laborales; cuarto acerca de la globalización; quinto, acuerdos y tratados, y sexto logística.
\end{abstract}

Palabras clave: relacionamiento económico, negocios internacionales, investigación, tendencias, clúster

Fecha de recibido: 15 enero de 2018 Fecha de aprobado: 8 de noviembre de 2018

Como citar: Valbuena, P. y Montenegro, Y. (2019). Los negocios internacionales y el relacionamiento económico: una revisión de la literatura. Revista Facultad de Ciencias Económicas, 27(1), 185-200. rev. fac.cienc.econ. doi: https://doi.org/10.18359/rfce.3233

JEL: F10, F23, F29.

* Artículo de revisión.

a Doctor en Estudios Sociales, máster en Historia y Economista. Profesor Asociado de la Facultad de Ciencias Económicas y Administrativas de la Universidad El Bosque, Bogotá, Colombia. Correo electrónico: valbuenapedro@unbosque.edu.co, pedronelvalbuena@yahoo.com

b Abogada, máster en Globalización, Comercio Internacional y Mercados Emergentes, y doctora en Derecho. Profesora asociada e investigadora de la Facultad de Ciencias Económicas y Administrativas de la Universidad El Bosque, Bogotá, Colombia. Correo electrónico: ymontegro@unbosque.edu.co 


\title{
International Business and Economic Relations: A Literature Review
}

\begin{abstract}
This paper discusses general research trends in economic relations and international business, based on the review of scientific literature. The objective hereof was to examine subject backgrounds from the scientific publications included in the Scopus database. Data mining and content analysis techniques were the methods used to identify clusters in the field of study, in an observation window covering nearly three decades of publications, identifying core and hierarchical topics. The article seeks to provide an overview of the current state of knowledge production, thus allowing academics and researchers to guide future research within the field of international business. The six most important groups were the following: company studies and expansion processes; productivity and export behavior; labor relations; globalization; agreements and treaties, and logistics.
\end{abstract}

Keywords: economic relationship, international business, research, trends, cluster

\section{Os Negócios Internacionais e Relações Econômicas: Uma Revisão da Literatura}

Resumo: Este artigo apresenta tendências gerais de pesquisa em relações econômicas e negócios internacionais, com base na revisão da literatura científica. O objetivo desse instrumento foi examinar os temas de origens das publicações científicas incluídas na base de dados Scopus. Como método, técnicas de mineração de dados e análise de conteúdo foram utilizadas para identificar agrupamentos no campo de estudo, em uma janela de observação que abrange quase três décadas de publicações, identificando tópicos centrais e hierárquicos. O objetivo deste artigo é mostrar uma visão geral do estado atual da produção de conhecimento que permite a acadêmicos e pesquisadores orientar futuras pesquisas no campo dos negócios internacionais. Os seis grupos mais importantes foram os seguintes: estudos sobre a empresa e processos de expansão; comportamento de produtividade e exportação; relações laborais; globalização; acordos e tratados, e logística.

Palavras-chave: relacionamento econômico, negócios internacionais, pesquisa, tendências, agrupamentos 


\section{Introducción}

Los estudios vinculados con el estado de la investigación en negocios internacionales en los últimos cinco años, publicados en Scopus, han sido escasos y se han dado en campos particulares que no dan una visión integral de las tendencias en el área. Se resalta el trabajo de Lee y Rha (2018), quienes se enfocaron en estudiar las tendencias de investigación en el sector de los servicios en el periodo de 2007-2017. Por otra parte, el estudio de Iammarino, McCann y Ortega-Argilés (2018) que relacionó tendencias correspondientes a multinacionales, competitividad y ciudades globales durante las últimas cinco décadas. En otro campo, está el estudio de Naray y Bezençon (2017), el cual usó la categoría de diplomacia comercial para verificar tendencias de investigación en el periodo 19602014. Se suman Albort-Moran y Ribeiro-Soriano (2016), que revisan las tendencias de la literatura sobre incubadoras de empresas. Finalmente, White, Guldiken, Hemphill, He y Sharifi (2016) se enfocaron en abordar el tema de la gestión estratégica internacional desde 2000 hasta 2013. Es de anotar que Valbuena y Montenegro (2017) buscaron identificar de manera amplia las principales áreas de investigación en torno a los negocios internacionales, lo que derivó en un estudio específico (Montenegro y Valbuena, 2018) que propuso una revisión general del relacionamiento jurídico en los negocios internacionales. Este artículo en particular está enfocado en el relacionamiento económico.

El escrito propone un aporte al campo de estudios enfocados al relacionamiento económico en los negocios internacionales. Así, considerando que el estado actual de la literatura evidencia la existencia de revisiones en temas específicos en este aspecto, es necesario elaborar una revisión dirigida a establecer de manera general algunas tendencias temáticas de investigación. En este sentido, el propósito del presente artículo es identificar inclinaciones de las investigaciones que de alguna manera delimitan futuras indagaciones en el campo de estudio.

Se encontró una variedad temática de las investigaciones vinculadas al relacionamiento económico, integrado a los negocios internacionales, lo que mostró un gran impacto y prevalencia de esta categoría. En este estudio se utilizaron técnicas de minería de datos y análisis de contenido para identificar las tendencias temáticas. El eje central que permitió la sistematización fue la identificación de clúster, a partir de la revisión de la literatura científica, delimitado por la categoría central: relacionamiento económico, en una ventana de observación que abarcó publicaciones científicas entre 1982 y 2017. Sin duda, este panorama descriptivo señala una visión de conjunto para los profesionales y académicos interesados en estos temas. El análisis mostró agrupaciones de la literatura del relacionamiento económico en los negocios internacionales, a partir de autores, países y publicaciones más representativos del campo de investigación. Se tuvieron en cuenta para describir los resultados categorías asociadas a los clústeres de mayor significancia, a saber: en primer lugar, los procesos de expansión y estudios sobre la firma; en segundo lugar, aspectos vinculados con la productividad y el comportamiento exportador; en tercer lugar, dinámicas del trabajo; en cuarto lugar, factores asociados a la globalización; en quinto lugar, aspecto relacionado con los acuerdos y tratados, y por último, lo relacionado con la logística y la sostenibilidad. La relevancia y la contribución científica de este artículo basan su aporte en la agrupación hecha con el análisis bibliométrico, en aspectos centrales como la internacionalización de la empresa, las ventajas comparativas, competitividad, creación e innovación y globalización. Contribuye, además, a la identificación de factores para la exploración de futuras investigaciones que den repuesta a los vacíos del conocimiento en el campo de estudio. El artículo se estructura, primero, con la presentación de la metodología, luego, con los resultados y finalmente con la discusión y las conclusiones.

\section{Método}

\section{Selección de casos}

Se realizó una búsqueda en Scopus, considerada la base de datos de citas y resúmenes más grande de la literatura científica, la cual ofrece herramientas para el análisis y la visualización de la investigación. 


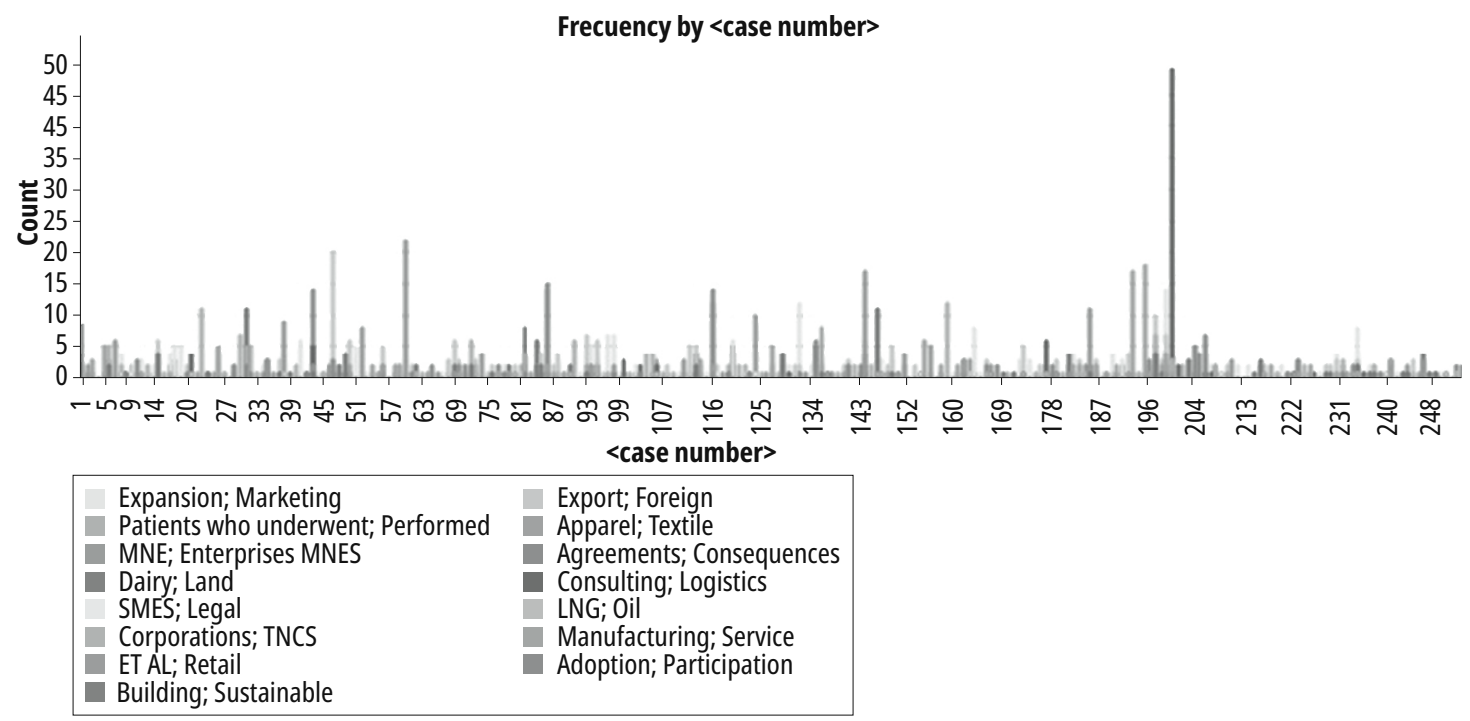

Figura 1. Temas centrales de relacionamiento económico en los negocios internacionales.

Fuente: elaboración propia.

Se introdujeron en la búsqueda las categorías Economic relationship AND international business. Los resultados iniciales de búsqueda fueron filtrados temáticamente para seleccionar solo artículos publicados en revistas científicas relacionados. Además, se excluyeron otros tipos de documentos como conference papers, book chapters, books, editorials and reviews. El proceso de filtrado redujo el número inicial de resultados de 1300 artículos. Luego, se definió una ventana de observación entre 1982-2017, que incluyó una selección final de 250 artículos. Se conformó una base de datos con los títulos, abstracts, autores, revista, país y año de publicación de los artículos seleccionados, que luego fueron tratados en el programa wordstat 7.1.

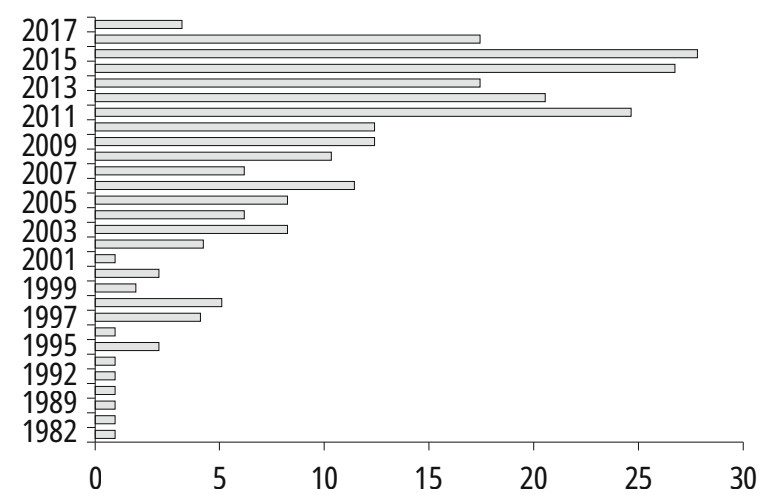

Figura 2. Crecimiento de las publicaciones. Fuente: elaboración propia, a partir de data base Scopus.
En consideración con la ventana de observación, el lector encontrará una centralidad de la exposición de los artículos científicos en un rango significativo en los últimos cinco años, las publicaciones elaboradas en los demás años son tratados de manera marginal y se ilustran como factor de determinación de los clústeres.

\section{Análisis de datos}

Se elaboró un análisis de contenido dirigido a identificar tendencias temáticas en el campo de investigación de los negocios internacionales. Para asegurar la confiabilidad del análisis de contenido, se combinaron técnicas manuales y asistidas por computador (minería de datos). En primer lugar, se analizaron las tendencias generales en el campo de la investigación en negocios internacionales; en particular, se delimitó a artículos científicos en la ventana de tiempo de observación. En segundo lugar, se conformó un listado de palabras clave a partir de un análisis de frecuencias (palabras repetidas) en los abstracts de los artículos escogidos. El análisis de frecuencias se realizó usando el software Wordstat 7.1 (Provalis Research, 2014). Las palabras con menos de 100 ocurrencias fueron descartadas del listado. Luego, se realizó una limpieza manual sobre la lista de palabras clave eliminando los términos conceptualmente irrelevantes; 
después, se hizo un análisis factorial exploratorio (método de rotación Varimax) sobre los datos de frecuencia de palabras para revelar la estructura temática latente de los abstracts. Las palabras con pesos primarios (eigen values) menores a 0,4 fueron excluidas del proceso de extracción. En tercer lugar, se revisaron de forma manual los abstracts clasificados en cada factor y se llevó a cabo una descripción cualitativa de su contenido, mediante el método de saturación, a partir de los clústeres señalados en la tabla 1. En consecuencia, se identificaron seis clústeres, el criterio de jerarquización se definió a partir de su peso (porcentaje de casos); además, al examinar factorialmente (eigenvalue), estos también tienen mayor cohesión entre sus descriptores (keywords). Es de resaltar que el método de selección de la muestra puede advertir una limitación en la investigación, al descartar artículos basados en otras categorías como impacto de los autores o de las publicaciones, incluso al obviar la selección de otras bases de datos, que contienen publicaciones relevantes en el campo.

Tabla 1. Tendencias de investigación en relacionamiento económico

\begin{tabular}{|c|c|c|c|c|c|c|c|}
\hline 을 & $\underline{z}$ & 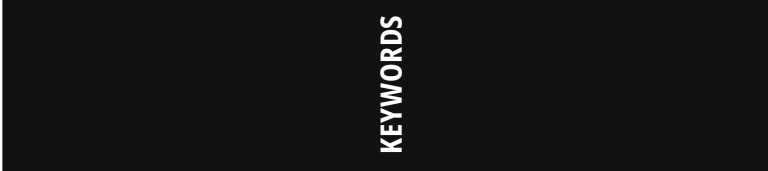 & 岂 & $\underset{\mathrm{a}}{\stackrel{\circ}{\mathrm{s}}}$ & 品 & 㞾 & $\begin{array}{l}\text { 㟧 } \\
\vdots \\
0\end{array}$ \\
\hline 1 & EXPANSION; FIRM & $\begin{array}{l}\text { EXPANSION; FIRMS; MARKETING; INTERNATIONALIZATION; } \\
\text { OVERSEAS; INDUSTRIES; COMPANIES; INDUSTRY; STRATEGIES; } \\
\text { FOREIGN; MARKET; SOFTWARE; MARKETS; EXPORTERS }\end{array}$ & 3,27 & 1,66 & 490 & 179 & 70,75 \\
\hline 2 & $\begin{array}{l}\text { AGRICULTURAL; } \\
\text { PRODUCTIVITY }\end{array}$ & $\begin{array}{l}\text { AGRICULTURAL; PRODUCTIVITY; PRODUCTION; UNION; EXPORT; } \\
\text { FOOD; DAIRY; PRODUCERS; TRADE; DUE; EXPORTS; SUPPLY }\end{array}$ & 3,12 & 1,31 & 278 & 132 & 52,17 \\
\hline 3 & ECONOMY; LABOR & $\begin{array}{l}\text { ECONOMY; LABOR; INVESTMENT; FOREIGN; CAPITAL; SHARE; } \\
\text { EXPORT; INVESTORS }\end{array}$ & 2,74 & 1,33 & 242 & 120 & 47,43 \\
\hline 4 & INTRA, GLOBAL & $\begin{array}{l}\text { APPAREL; INTRA; TEXTILE; NATIONS; SECTORS; INDEX; } \\
\text { EXPORTING; COMPLEX; INCOME; SPECIALIZATION; REVEALED; } \\
\text { INTENSIVE; MODELS; DETERMINE; PRODUCT }\end{array}$ & 4,3 & 1,83 & 199 & 103 & 40,71 \\
\hline 5 & $\begin{array}{l}\text { AGREEMENTS; } \\
\text { SECTION }\end{array}$ & $\begin{array}{l}\text { AGREEMENTS; SECTION; CONSEQUENCES; BARRIERS; } \\
\text { LIBERALIZATION; FLOWS; INVESTMENTS; WTO; DISCUSSED; FREE; } \\
\text { IMPORTS; EFFORTS; CONCEPTS; ADDRESS; WORKERS }\end{array}$ & 4,09 & 1,83 & 169 & 98 & 38,74 \\
\hline 6 & $\begin{array}{l}\text { CONSULTING; } \\
\text { LOGISTICS }\end{array}$ & $\begin{array}{l}\text { CONSULTING; LOGISTICS; CLUSTERS; COMMERCIAL; CO; SMES; } \\
\text { CULTURAL; NETWORK; GOVERNANCE; BANKS; REGION; VALUES; } \\
\text { KNOWLEDGE; ACCOUNTING; INNOVATION }\end{array}$ & 19,05 & 1,72 & 156 & 98 & 38,74 \\
\hline 7 & $\begin{array}{l}\text { SOFTWARE; } \\
\text { SERVICE }\end{array}$ & $\begin{array}{l}\text { SOFTWARE; SERVICE; MANUFACTURING; SERVICES; } \\
\text { OUTSOURCING; ORGANIZATION; UNITED; SOURCING }\end{array}$ & 2,66 & 1,35 & 149 & 84 & 33,20 \\
\hline 8 & LNG; OIL & $\begin{array}{l}\text { LNG; OIL; ENERGY; TECHNOLOGIES; GAS; SUSTAINABLE; NATURAL; } \\
\text { PROCESSES; RESOURCES; ENVIRONMENTAL; ASSESSMENT }\end{array}$ & 3,91 & 1,46 & 134 & 77 & 30,43 \\
\hline 9 & $\begin{array}{l}\text { SUSTAINABILITY; } \\
\text { SOCIAL }\end{array}$ & $\begin{array}{l}\text { SUSTAINABILITY; SOCIAL; SUSTAINABLE; GLOBALIZATION; } \\
\text { EFFICIENCY; SMES; STRUCTURES; STANDARDS }\end{array}$ & 2,66 & 1,19 & 130 & 79 & 31,23 \\
\hline 10 & DAIRY; LAND & $\begin{array}{l}\text { DAIRY; LAND; GOVERNMENTS; CORPORATIONS; BANKS; } \\
\text { BUSINESSES; GOVERNMENT; MATERIALS; WESTERN; OIL }\end{array}$ & 2,8 & 1,34 & 125 & 74 & 29,25 \\
\hline 11 & $\begin{array}{l}\text { MNE; } \\
\text { ENTERPRISES } \\
\text { (MNES }\end{array}$ & $\begin{array}{l}\text { MNE; MNES; MULTINATIONAL; BOP; INSTITUTIONAL; IB; } \\
\text { INTERNALIZATION; ENTERPRISES }\end{array}$ & 3,82 & 1,41 & 113 & 68 & 26,88 \\
\hline 12 & $\begin{array}{l}\text { CARBON; } \\
\text { ADOPTION }\end{array}$ & $\begin{array}{l}\text { CARBON; ADOPTION; GAS; ENVIRONMENTAL; CLIMATE; TAX; } \\
\text { SYSTEMS; PARTICIPATION; POST }\end{array}$ & 2,9 & 1,51 & 94 & 62 & 24,51 \\
\hline 13 & RETAIL; RETAILERS & RETAIL; RETAILERS; JOB; LOGISTICS; ET; AL; POWER; BUSINESSES & 3,18 & 1,12 & 64 & 46 & 18,18 \\
\hline
\end{tabular}

Fuente: elaboración propia. 


\section{Resultados}

\section{Relacionamiento desde la perspectiva de la internacionalización de la empresa}

A partir de los datos, se puede señalar que la categoría de mayor relevancia es sin duda la internacionalización de la empresa. Aun así, situar el relacionamiento económico en esta perspectiva resulta difuso, dada la amplitud temática. Esto se evidenció, por ejemplo, al encontrar indagaciones enfocadas en los estudios de caso; Luiz, Stringfellow y Jefthas (2017) usaron las categorías internacionalización de la empresa y expansión de una empresa multinacional, mediante un estudio de caso en Sudáfrica. Los autores encontraron una relación directa entre expansión de las compañías y el aumento del riesgo en relación con dos variables: incertidumbre institucional y el riesgo país, factores que fueron sobrepuestos mediante la función de aprendizaje por la práctica, es decir, que el desarrollo del país y las condiciones institucionales del país de origen permitieron un exitoso proceso de expansión. Siguiendo el análisis de factores de riesgo, Thompson (2001) encontró para el caso de Hong Kong que el atractivo del entorno empresarial está directamente influenciado por la política gubernamental, aspecto que puede sobreestimarse a la hora de incursionar en este mercado.

Por otro lado, la internacionalización de la empresa, basada en un criterio de innovación y aprendizaje, se constituye en un matiz sobresaliente en el relacionamiento económico. Hay que anotar que Patel y Pavitt (1994) han enfatizado en la importancia de los patrones nacionales de especialización tecnológica que reflejan caminos acumulativos y localizados del aprendizaje tecnológico y que influyen fuertemente en los patrones de cambio estructural y en las ventajas comparativas en el comercio. Ahora bien, respecto a la relación entre aprendizaje de las empresas y la ampliación de las exportaciones, en el trabajo de Nguyen y Nguyen (2010) se encontró que el mantenimiento de relaciones a largo plazo con los clientes afianza el proceso de internacionalización y ampliación de mercados, siendo la calidad el criterio fundamental para ampliar las exportaciones. Al respecto, Entrup y Barth (2008) vincularon el término Business-Process-Reengineering (BPR) para describir el enfoque de la organización de procesos a lo largo de los requisitos del cliente, y así dieron cuenta de la calidad del producto como un factor determinante en el desarrollo de ventajas comparativas en el comercio internacional. Kundu y Renko (2005) afirmaron que el bajo nivel de internacionalización de las economías emergentes se debió en gran parte a la falta de demanda de productos o servicios en los respectivos países de origen. Por otra parte, para países con mayor desarrollo, Ruamsook, Russell y Thomchick (2009) advirtieron que las prioridades de mejora para las empresas estadounidenses manufactureras son la capacidad de producción del proveedor, la cultura y las prácticas empresariales, y la infraestructura de comunicación.

La internacionalización de la empresa resultó ser vinculada también a los procesos estructurales de transnacionalización económica. En este aspecto, sobresalen las investigaciones vinculadas a la expansión de las multinacionales (EMN); se encontró el estudio de Williamson (2014), quien concluyó que el proceso de internacionalización se está dando en un entorno altamente globalizado que redunda en un incremento del comercio internacional, también como un fuerte dinamismo de la inversión y flujos de conocimiento, así como cadenas de valor globales. Por su parte, Yeoh (2011) abordó el concepto de multinacionales emergentes mediante el estudio de las estrategias de internacionalización en dos empresas farmacéuticas indias, y concluyó que la adquisición (en lugar de nueva inversión) en el extranjero proporciona acceso a las redes de mercadeo establecidas, lo que aumenta las ventajas de propiedad de las empresas. Ahora bien, Regnér y Edman (2014) afirmaron que los factores de éxito de la EMN están dados por la innovación, el arbitraje, la evasión y adaptación, mediante el anclaje institucional y su papel de agencia dentro de las dinámicas de internacionalización. Por otro lado, Agmon y Messica (2009) señalaron que la inversión de fondos de capital privado en mercados emergentes se muestra como una nueva forma de inversión extranjera directa denominada inversión extranjera directa financiera (FFDI). 
En otra dirección, Derado (2011), a partir de estudios de caso en Croacia sobre internacionalización de la pequeña y mediana empresa, señaló que el apoyo a los servicios empresariales está dirigido principalmente a crear condiciones para el crecimiento y el desarrollo. En una fase más madura, sin embargo, la mejora de la competitividad internacional de las empresas locales resulta ser una herramienta más eficaz para la internacionalización a largo plazo de los negocios. Por otra parte, los estudios sobre pymes, como el de McNamara (2006), identificaron que al no tener acceso al entorno de aprendizaje de sus centros de producción en el extranjero, las pymes asiáticas a menudo siguen siendo fábricas extraterritoriales en lugar de ser empresas internacionales que participan en entornos globales de aprendizaje. Sobre este aspecto, en particular emergen estudios relacionados con los entornos globales de aprendizaje, asociados a la emulación de estrategias y estructuras legítimas de las empresas internacionales por parte de las organizaciones criminales transnacionales (Enderwick, 2009), como formas de innovación en actividades ilegales o de economía subterránea.

En las tendencias de investigación, resultó peculiar la atención de los estudios sobre la economía china. Un análisis del mercado de esta economía sugiere que las exportaciones de este país y su ventaja comparativa en los mercados internacionales no son afectadas de manera significativa por los mayores costos laborales locales y las regulaciones establecidas a través de aumentos en las normas de salario mínimo (Gan, Hernández y Ma, 2016). También, Martek y Chen (2016) señalaron en un estudio comparado que las empresas chinas basan su desempeño en el proceso de internacionalización a través de la búsqueda de fuentes de abastecimiento óptimas, el desarrollo de monopolios y la dominación del mercado, ofertas únicas diferenciadas, o mediante la integración con proveedores, además de adquisiciones intensivas en tecnología off-shore. Por su parte, Renchu (1999) examinó las principales diferencias entre las empresas manufactureras chinas y japonesas, y presentó sugerencias sobre la estrategia de desarrollo de la industria manufacturera china. Además, una investigación de Malik (2012) reveló que las empresas que habían entrado en los mercados chinos fueron capaces de desviar algunas de las pérdidas causadas por la desaceleración global. Ahora bien, Wilson y Brennan (2003) consideraron que el ingreso de empresas internacionales al mercado chino depende del tamaño de la empresa, la experiencia en China, el tipo de industria y las condiciones en el macroambiente. Desde otra perspectiva, Eng y Lin (1996) señalaron que la manipulación efectiva de la acción estatal es clave para el éxito económico de las inversiones extranjeras en empresas en la China. A su vez, Ancharaz y Tandrayen-Ragoobur (2013) han señalado la falta de penetración de las exportaciones a China a causa de factores sistémicos, como la falta de complementariedad comercial, la escasa competitividad de las exportaciones, el sesgo del mercado de exportación, entre otros.

\section{Emergencia de nuevo estatus de las ventajas comparativas}

El relacionamiento económico visto desde la categoría de ventaja comparativa señala una convergencia hacia la revalorización del concepto en el contexto de una economía global donde ha prevalecido la noción de ventaja competitiva. Lo anterior es ratificado por la revisión de Luo y Zhang (2016), los cuales encontraron que las nuevas perspectivas de investigación están en el estudio de ventajas comparativas además de las competitivas. Por ejemplo, Kuznetsov (2016) vinculó este aspecto al análisis de las relaciones mutuas de inversión extranjera directa entre Rusia y diversos países. Wei y Joyce (2016), a través de un estudio multicaso, trataron aspectos concernientes a las cadenas de valor, aplicados al sector de la floricultura no corporativa en Fiji, Islas Salomón y Papua Nueva Guinea. Esto autores encontraron que para obtener una ventaja comparativa en el mercado se debe ofrecer: primero, productos únicos o diferenciados; segundo, precios competitivos a través de la eficiencia operativa, y tercero, buenos servicios a través de la creación de relaciones con segmentos de nicho de mercado. En consecuencia, se advierte prevalencia de estudios de ventajas comparativas en el relacionamiento económico, con diversos matices y alcances. Por ejemplo, Hung y Chou 
(2014) indagaron acerca de las influencias culturales sobre la adopción del comercio móvil basado en los casos comparativos de intercambio comercial entre Taiwán y Malasia. Adicionalmente, Taneja, Mehra, Mukherjee, Bimal, y Dayal, (2015) estudiaron la economía de la India y Pakistán, para establecer una potencial relación comercial, a través de la ventaja comparativa revelada (RCA). En el contexto de los estudios de caso sobre economías nacionales, sobresale el de Lemoine (2013), quien llegó a la conclusión de que los exportadores chinos deben pasar de la competitividad de los precios a la mejora de la calidad para crear nuevas ventajas comparativas.

Los trabajos de Kilduff y Chi (2006), por su parte, evaluaron los patrones de ventaja comparativa en los principales países exportadores de textiles y prendas de vestir. Javalgi, Gross y Granot (2011) examinaron los servicios empresariales intensivos en conocimiento (KIBS) en mercados emergentes para el análisis de ventajas competitivas, señalando que los países con mayor desarrollo en este aspecto son China, India, Brasil, Rusia, México, Turquía e Indonesia. Además, Avkiran (2017) abordó la categoría multiple stakeholder perspective (MSP), para hacer una estimación comparativa del desempeño bancario, en socios comerciales de Japón, China y Australia.

Por otro lado, se hallaron estudios generales acerca de las ventajas comparativas y su importancia en el comercio internacional basado en el enfoque de sostenibilidad y responsabilidad social, como el de Banerjee, Chaudhari, Salunkhe y Ravishankar (2013), quienes examinaron su relación con la sostenibilidad, enfocado al desarrollo del sector del transporte en India. También, Wysokińska y Witkowska (2005) exploraron los aspectos medioambientales, especialmente las estrategias de protección del medio ambiente aplicadas por los inversores extranjeros, a partir de una encuesta a 286 empresas polacas. Palekhova (2016) indagó acerca de los estándares de responsabilidad social en el campo del desarrollo sostenible para mejorar la competitividad de las empresas industriales ucranianas en el marco de la globalización. Por su parte, Andriani y Herrmann-Pillath (2015) estudiaron las capacidades transaccionales creadas por la acción emprendedora, en relación con el desarrollo de ventajas comparativas, a partir de un estudio de caso en la producción de café en Brasil. En la perspectiva de Harzing y Giroud (2014), las empresas internacionalmente activas deben capitalizar sus ventajas geográficas para consolidar sus ventajas comparativas; esto también lo menciona Audretsch (1998), y está asociado a la posición geográfica y la actividad innovadora, funcional y al crecimiento de los países como al de las firmas. En adición, Ahmed y Rock (2012), al estudiar empresas chilenas internacionales, sugirieron que las empresas de países pequeños, dotadas de ventajas comparativas basadas en recursos naturales, pueden penetrar exitosamente en los mercados de exportación. En particular, Yamori (1997) elaboró un estudio que buscó examinar el papel de los bancos en la internacionalización de las empresas japonesas señalando un notable liderazgo de los bancos japoneses en la internacionalización de las manufacturas de este país con destino a economías en Asia y Oceanía; demostró también que los bancos japoneses tienen una ventaja comparativa frente a los competidores del país anfitrión en estas regiones. Hay que anotar que Norberg-Hodge (2006) advirtió los problemas de una inadecuada política comercial basada en la ventaja comparativa y arguyó que esta aún guía la planificación del Gobierno y la toma de decisiones está en el corazón del dogma del libre comercio. A su vez, Templin (2010) examinó los marcos proteccionistas de Estados Unidos y China, considerándolos un obstáculo para el libre comercio; en ese sentido, la ventaja comparativa se daría como una falacia en el plano real del comercio global.

Asimismo, la relación entre ventajas comparativas e internacionalización de las empresas hace parte del inventario de tendencias. Por ejemplo, en cuanto a la instalación de plantas, Demeter y Szász (2014) afirmaron que estas pueden basarse en ventajas comparativas basadas en la localización, tales como mercados, oportunidades de producción de bajo costo o acceso a habilidades y conocimientos. De igual forma, Laguna (2004) relacionó el factor de localización, como determinante en el desarrollo de ventajas comparativas de las multinacionales, para el caso del sector del petróleo en México. 


\section{Competitividad, creación e innovación}

En función del marketing global, Saraswat (2012) identificó problemas gerenciales de empresas de la India, relacionados con el uso de la tecnología, asociados a infraestructura, regulación y restricciones prácticas de marketing del sitio en internet, formación y cultura, y restricciones financieras. Vrana y Zafiropoulos (2009) examinaron los asuntos del marketing en el turismo en países mediterráneos de Europa, basados en la interactividad, navegación y funcionalidad. Por otra parte, está el trabajo de Yang, Sonmez, Li y Duan (2015), que examinaron la evaluación del desempeño de la marca (satisfacción, lealtad y éxito percibido) asociado a ventajas y desventajas comparativas de las marcas locales y extranjeras en el ámbito internacional, basado en el uso de los portales web. Por su parte Sharif (1997) señaló que la competitividad tecnológica también brinda oportunidades a las empresas en los mercados globales, si están adecuadamente preparadas para desarrollar la ventaja comparativa tecnológica.

En las investigaciones acerca de logística, se encontraron los trabajos de Ruamsook et al. (2009), autores que estudiaron las fuentes de suministro en los países desarrollados e indicaron que las cuestiones que deben ser prioritarias para mejorar en el ámbito de la logística internacional son la capacidad de producción del proveedor, la cultura, las prácticas empresariales y la infraestructura de comunicación. Con esto se relaciona el trabajo de Myers, Fawcett y Smith (2002), basados en un estudio de caso, mediante entrevistas a gerentes de empresas manufactureras en Estados Unidos, cuyo mercado es América Latina y el Caribe, enfocados en la búsqueda de relaciones entre el desempeño logístico, de la manufactura y de la empresa.

Por otra parte, en relación con los análisis estratégicos, se identificaron estudios enfocados en el análisis de estrategias de cadenas de valor (Brautigam, Weis y Tang, 2018) y estrategias de negocios (Ben-Amor, Aguayo y Miguel-Gómez, 2015); en este se contemplaron factores como el acondicionamiento, nuevos tratamientos no químicos, embalaje, apertura de nuevos mercados, nuevos canales de distribución; también, estrategias de responsabilidad social (Li, Toppinen y Lantta, 2016); estrategias de posición de los mercados (González, Flores, Gil-Lafuente, Flores, 2015); estrategias verdes para mejorar su competitividad (Wang, Li y Mao, 2013); estrategias de redes (Tsang, 2006, y estrategias para mejorar la gestión e integración de los recursos de los sistemas de información (Selig, 1982).

En cuanto a la productividad, estudios como los de Kulubekova, Viglione, Busurmanov (2016) se enfocaron en analizar la creación de nuevas corporaciones transnacionales y su contribución al aumento de la competitividad global de la Unión Económica Eurasiática. En este sentido, Kyselová (2014), en relación con la competitividad de la Unión Europea, señaló que la tendencia indica que las inversiones en innovación y en tecnologías son claves en el proceso actual de integración económica y de expansión de los mercados, redefiniendo las cadenas mundiales de valor. De igual manera, Parausic, Potrebic, y Simonovic (2013) advirtieron que el incremento de la productividad en empresas con niveles de internacionalización está en función del aumento de las actividades de investigación y desarrollo, la conformación de asociaciones y agrupaciones, y la consolidación de un entorno empresarial microeconómico estimulante para las empresas. Además, Bond (2013) encontró que un grado favorable de éxito para firmas internacionales es el aprovechamiento de contratos una vez se ha insertado en el mercado internacional (relational contracts), es decir, mediante la repetición, aprendizaje y experiencia que dejan estos; es como una forma de consolidar una ventaja comparativa. Los trabajos que relacionan globalización y productividad están vinculados con la dinámica de producción de software; por ejemplo, Rajapriya, Kumar y Krishnan (2017) identificaron focos internacionales de Business Process Outsourcing (BPO) que han surgido en 1) India, ingeniería y técnica; 2) China, fabricación y técnica; 3) México, fabricación; 4) Estados Unidos, análisis y creatividad, y 5) Filipinas, administración. 


\section{Globalización}

Los aspectos concernientes a la globalización suelen ser recurrentes, por ejemplo, factores económicos y políticos junto con las perspectivas tecnológicas hacia el crecimiento de las exportaciones y la competitividad entre los países en desarrollo (Rajagopal, 2009). A su vez, Ushakov y Arkhipov (2013) abordan aspectos relacionados con la competitividad y eficacia de los sistemas empresariales urbanos en una economía globalizada. Sobre formación de capital humano, Koch (2007) afirmó que para Estados Unidos una condición diferencial con la dinámica de crecimiento de China —país que basa su crecimiento en bajos costos laboralesconsiste en generar cambios estructurales en la economía, el movimiento gradual de la cadena de valor de la producción y la política gubernamental para aumentar el capital humano, desarrollar el interior del país y disminuir la corrupción. En otro sentido, Cottier (2015) consideró un cambio de la liberalización del comercio hacia la regulación del comercio como papel central de la Organización Mundial del Comercio, o lo que se denomina la nueva gobernanza mundial (Kellow, 2012). En relación con la categoría outsourcing, Dash (2006) indicó que, para la subcontratación de servicios, la definición de capital humano debe restringirse a los estudiantes secundarios y particularmente a terciarios más que a la alfabetización, dado que para el comercio internacional, en este aspecto, debe considerarse la ventaja comparativa absoluta y no la relativa.

Por otro lado, el relacionamiento económico ha estado vinculado con los fenómenos del trabajo y su reestructuración en la internacionalización de la economía. Al respecto, Jensen (2016) demostró una fuerte correlación entre el producto interno bruto (PIB) per cápita y la proporción de servicios empresariales en el PIB, con un fuerte impacto en el empleo en países como Estados Unidos. En cuanto a la relación trabajo y competitividad, Contractor, Kumar y Dhanaraj (2015) señalaron los inconvenientes, como la legislación laboral, la inadecuada infraestructura y la participación de las mujeres para el caso de la economía de la India. Por su parte, Silvia (2006) señaló que los trabajadores poco calificados serán cada vez más relegados, a menos que mejoren sus habilidades en una economía global cada vez más competitiva. Además, Toulmin (1999) manifestó la tendencia de recortes excesivos en los sistemas de protección y seguridad social, expresiones negativas de la liberalización de mercados. Winters, Walmsley, Wang y Grynberg (2003) manifiestan que sería factible que se generaran rendimientos potencialmente grandes si se permitiera a los trabajadores medianos y menos calificados, que son relativamente abundantes en los países en desarrollo, prestar sus servicios en los países desarrollados. A su vez, Cambra-Fierro y Ruiz-Benítez (2011), en un estudio de caso en España, concluyen que la cultura es un factor decisivo en la implementación de prácticas sostenibles.

Finalmente, de acuerdo con la tabla 1, se sugiere un campo de investigación significativo en la categoría sostenibilidad, pero en menor escala, en especial, estudios relacionados con responsabilidad social y sostenibilidad. En este aspecto, Zostautiene, Susniene, Purvinis, Sargunas y Puckyte, (2016) examinaron las tendencias de la responsabilidad social corporativa (RSE) y revelan la manifestación de sus prácticas en las multinacionales extranjeras y nacionales que operan en Lituania. También, Walter y Segerstedt (2012) evaluaron el comercio internacional de biocombustibles, prestando especial atención a los países africanos, que tienen un potencial razonable para la producción de biocombustibles; así, concluyeron las aceptables perspectivas de desarrollo del sector, en ocasión de la aplicación real del comercio justo. De igual forma, Williams et al. (2015), enfocados en la industria de gas natural a partir de las cadenas de valor internacionales, formularon la necesidad de un mayor desarrollo de datos sobre las emisiones y una mayor transparencia de las características de las emisiones para las tecnologías alternativas, que promuevan el desarrollo tecnológico para mejorar el desempeño ambiental. Por otro lado, se encontró el estudio de Cary y Roberts (2011), que analizo la eficacia de los programas apoyados por el gobierno para fomentar una mejor gestión de los sistemas de tierras y agua. Los aspectos de gobernanza también hacen presencia en este aspecto, por ejemplo, aquellos relacionados con la gestión transnacional 
y la política pública para el desarrollo económico (Pearce, Xin, Xu y Rao, 2011).

\section{Discusión}

La categoría que propone el presente artículo, de relacionamiento económico, da cuanta de las vinculaciones entre economías - a nivel de las naciones-, empresas u organizaciones, basada en principios y valores de interacción e interdependencia, bajo un orden de intereses estratégicos y de reciprocidad relativa en la integración económica, que en cada momento y tiempo se da desde un nivel de restructuración productiva y comercial.

El relacionamiento económico integra y jerarquiza diversos sistemas de producción en coexistencia de mercados imperfectos y de mercados libres que, en el momento actual, son estructurados a partir de procesos de transnacionalización de la economía y fuertes cambios políticos y de (des)regulación económica internacional. En este sentido, al vincular el relacionamiento económico y los negocios internacionales, sobresalen tendencias de investigación que se pueden aglutinar en los siguientes ejes temáticos:

a) Internacionalización de la empresa.

b) Ventajas comparativas.

c) Competitividad, creación e innovación.

d) Globalización.

Aunque esta organización es arbitraria, obedece a los hallazgos de los clústeres; además estos interactúan en distintos niveles, lo que llevó a encontrar en el estudio aspectos como: procesos relacionados con la innovación y la gestión logística, el diseño y diferenciación de productos, pautas de éxito en el proceso de internacionalización (Moroni, Arruda, Bezerra y Laila, 2018).

Emergen aspectos como la expansión de las compañías o aquellos asociados al emprendimiento internacional y la determinación de características empresariales y nueva empresa internacional (Kowalik, Danik y Sikora, 2017); asimismo, los aspectos relacionados con factores de riesgo y procesos de mitigación a marcos regulativos, cambios políticos y procesos sancionatorios a la empresa internacional. Por ejemplo, Ankudinov,
Ibragimov y Lebedev (2017) estudiaron la experiencia de las empresas rusas en el proceso de integración en el mercado global y encontraron factores de éxito asociados principalmente al flujo de la inversión extranjera directa, comercio de materias primas, alianzas internacionales $y$ suministro extensivo de servicios logísticos y estratégicos. Hacen parte de los ejes temáticos los patrones nacionales de especialización tecnológica, las prácticas de gestión, la innovación y el capital humano (Skorupinska, 2017).

Después de encontrar en esta revisión el auge de los estudios vinculados a las ventajas comparativas, es importante resaltar aquellos que se enfocan en aspectos como la cooperación en el comercio internacional y condiciones de competitividad internacional (Torok y Jambor, 2016). La interacción con los otros ejes temáticos se da en aspectos como el aprendizaje de las empresas para las exportaciones, la ampliación de mercados y diversificación de las exportaciones.

En el relacionamiento económico, son funcionales las dinámicas de las multinacionales (EMN), a saber, los determinantes de la expansión de una empresa multinacional (Malik y Velan, 2017) y cadenas de valor globales. Incluso incursionan categorías de interesante exploración como las multinacionales emergentes, en algunos estudios asociados a estrategias de integración, construcción de redes de producción, distribución, logística, etc., así como las estrategias globales de adquisición y expansión global (Bala Subrahmanya, 2007).

Tienen especial relevancia los estudios asociados al comportamiento, determinantes y dinámicas de la inversión extranjera directa, entre los cuales se destacan las características de localización, el papel de la política pública y comercial de los países, los factores de atracción y retención de la inversión extranjera, así como las motivaciones para desarrollar IED por parte de las multinacionales (Annan-Diab y Filippaios, 2017).

Finalmente, de manera especial se estudian aspectos relacionados con las influencias e interacciones interculturales, también formas de percepción y de construcción de identidad, y estudios aplicados a las dinámicas del trabajo 
(Rozkwitalska, Chmielecki, Przytula, Sulkowski y Basinska, 2017).

Adicionalmente, se encuentran estudios asociados a las dinámicas de inversión de fondos de capital, como FFDI. Son recurrentes los estudios empíricos que establecen correlaciones entre la cuenta corriente, las acciones, tipos de cambio, precios, cuenta de capital, así como la incidencia de las políticas monetarias y comportamiento de los fondos mutuos en los mercados de renta variable (Lin y Fu, 2016) o riesgo macroeconómico (Bali, Brown y Caglayan, 2016). Finalmente, puede apreciarse una dispersión de estudios que van desde el desempeño bancario o los estudios relacionados con el sector del transporte y de infraestructura; otros estudios tratan aspectos medioambientales, estrategias de protección.

Categorías como sostenibilidad, responsabilidad social y sostenibilidad, son usadas en los estudios a partir de conceptos como el de RSE en las multinacionales, por ejemplo, investigaciones acerca de los efectos de las transgresiones de las EMN y efecto sobre los consumidores (Chang, Jang, Lee Lee y Chang, 2017). Incluso se encontraron estudios de RSE en la gestión de los sistemas de tierras y agua, gobernanza, gestión transnacional y la política pública para el desarrollo económico, aunque fueron tópicos marginales dentro de las tendencias.

Siendo un foco central los estudios relacionados con las economías emergentes en el relacionamiento económico, las tendencias se enfocan en los estudios vinculados a la cultura y las prácticas empresariales: infraestructura, logísticas y comunicaciones; también, entornos de aprendizaje, centros de producción en el extranjero; dinámicas de pymes, política comercial. Todas son investigaciones que trataron el factor tecnológico, asociado a infraestructura, formación de capital humano y relacionamientos culturales, institucionales, de transparencia y corrupción; de igual manera, dinámicas de los mercados reales y financieros. Estudios más especializados se ocupan del marketing internacional, del desempeño de la marca (satisfacción, lealtad y éxito percibido), de la regulación y las restricciones prácticas de marketing del sitio en internet. A esto se suman aspectos de mercado internacional (relational contracts) y cadenas de valor. Marginalmente, algunos estudios críticos se han desarrollado en torno a la liberalización del comercio y regulación, fenómenos y dinámicas del trabajo y migración, en el marco de la reestructuración económica global, que se constituyen en vacíos de conocimiento en estos aspectos.

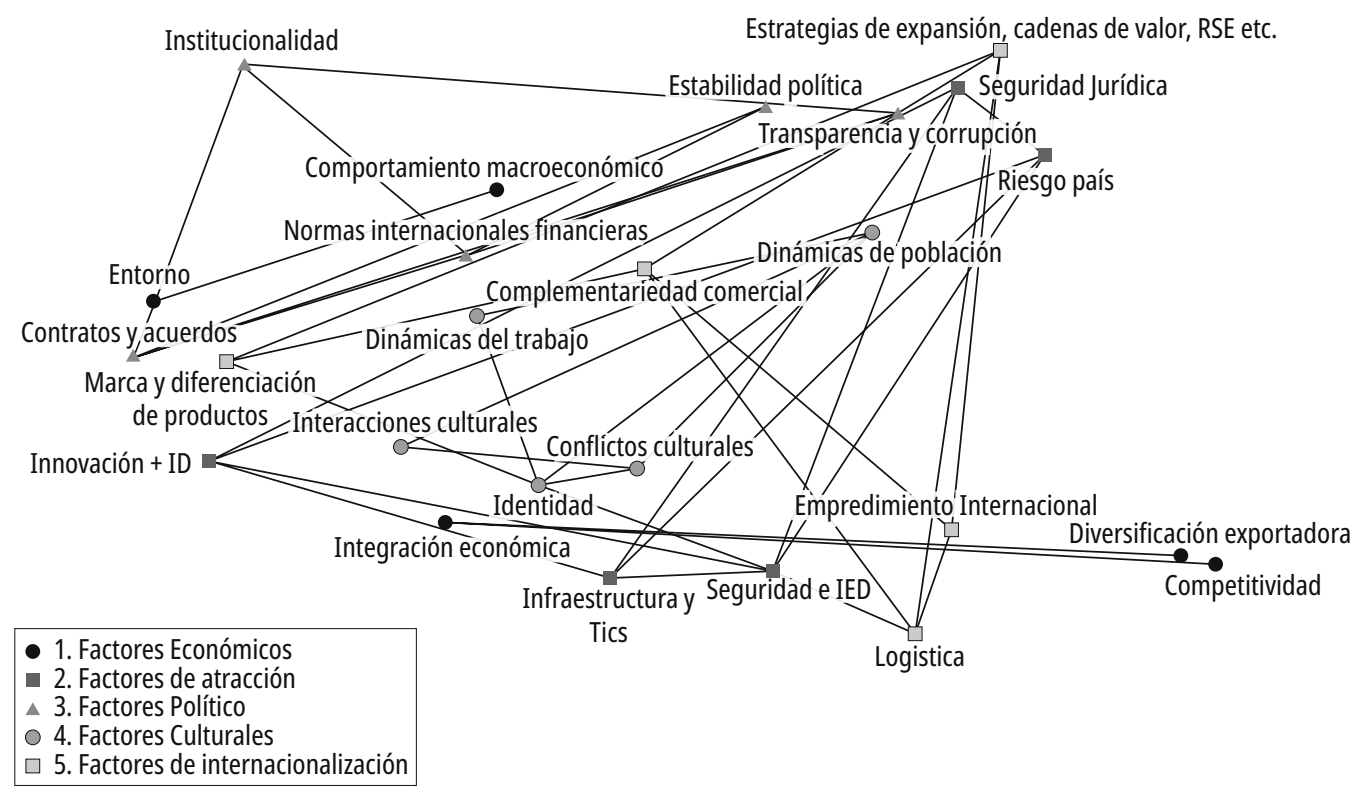

Figura 3. Factores y subgrupos de investigación.

Fuente: elaboración de los autores. 
Por último, se evidenció la existencia de investigaciones enfocadas en el análisis estratégico, de las cuales se encontraron estrategias de cadenas de valor, estrategias de negocios, estrategias de responsabilidad social, estrategias de posición de los mercados y estrategias verdes para mejorar su competitividad, y estrategias para mejorar la gestión e integración de los recursos de los sistemas de información, principalmente.

En consecuencia, las investigaciones que sugieren tratar integralmente el relacionamiento económico en los estudios de los negocios internacionales podrían vincular los factores que se describen en la figura 3, en gran parte, sustraídos de las agrupaciones temáticas que en esta revisión se han encontrado. El peso de cada factor depende de las intencionalidades y alcances de las apuestas investigativas y su aporte a la construcción de conocimiento en los negocios internacionales; a la vez, su tratamiento integral propone una potencial vinculación de la disciplina hacía otros campos de saber.

\section{Conclusiones}

Se tuvieron en cuenta para los resultados categorías asociadas a los clústeres de mayor significancia y se agrupó su descripción a partir de cuatro ejes temáticos: internacionalización de la empresa, ventajas comparativas, competitividad, creación e innovación, y globalización.

Ahora bien, los clústeres señalaron categorías temáticas de gran centralidad, asociadas por ejemplo a la internacionalización de la empresa y vinculadas al primer clúster: la expansión de la firma, economías emergentes, empresas multinacionales, pymes, etc.; dentro de los procesos de expansión se indicaron como temáticas recurrentes en la literatura como los factores de riesgo, análisis de entorno y factores de éxito. Siendo los estudios de EMN importantes dentro de los estudios de relacionamiento económico en los negocios internacionales, además de los aspectos mencionados, se incluyen otros como el agenciamiento de las EMN, inversión extranjera directa, fondos de capital, desarrollo de ventajas competitivas, relacionamiento cultural, gestión y logística. Hacen parte de este conglomerado categorías asociadas al marketing y los análisis estratégicos de la firma.

Acerca de las dinámicas de las exportaciones, eje central del segundo clúster, se asocian al tamaño de las empresas, los mercados, la complementariedad comercial, la competitividad de las exportaciones, los tratados comerciales, entre otros. Es de resaltar que el relacionamiento económico en función de los estudios del trabajo en los negocios internacionales, se vincula a categorías como reestructuración productiva, del trabajo, productividad, capital humano, economía informal. En este clúster, las categorías emergentes dieron cuenta de la existencia de la globalización como un aspecto central por tratar en las revisiones examinadas, y se señalaron factores económicos y políticos, tecnológicos, competitividad, sistemas empresariales, cadenas de valor, marketing global, entre otros, que se discuten alrededor de procesos de regulación o liberación de los mercados. En la misma dirección, las ventajas competitivas juegan un papel transversal en estos estudios.

En los demás clústeres se evidencia una dispersión temática, la tendencia de estudios se vinculan a aspectos relacionados con los tratados comerciales, la apertura y liberalización económica. En aspectos como la logística se estudian temas relacionados con capacidad de producción, relacionamiento cultural, prácticas empresariales, infraestructura, comunicación, desempeño logístico. Finalmente, se abordaron temas relacionados con sostenibilidad y RSE, gestión ambiental, prácticas de producción limpia y estudios del desarrollo, entre otros.

Cabe resaltar que el relacionamiento económico esta mediado en gran parte por los estudios de ventajas comparativas. Es una categoría que con frecuencia es incluida de manera transversal en las agrupaciones temáticas que se presentaron en este artículo.

Limitaciones: este artículo señala un panorama general que podría servir como base para investigaciones futuras, en temas de relacionamiento económico en los negocios internacionales. Además, es necesario elaborar indagaciones en campos poco estudiados, por ejemplo, el de los estudios críticos en negocios internacionales 
y relacionamiento político, no solo para la construcción de conocimiento en los ámbito de la profesión del negociador internacional, sino también para las relaciones interdisciplinares, en especial aquellas centradas en la reflexión acerca del sujeto, la sociedad y en general de las tensiones de la cultura y la vida contemporánea, desde el paradigma de una continua desestructuración del orden global, nacional y local del mundo. En todo caso, los vacíos y focos de interés para futuras investigaciones están vinculados al estudio de factores económicos, de atracción, políticos, culturales y de internacionalización, que desde la perspectiva del relacionamiento económico dejan un campo prolífico de indagación para el ámbito de conocimiento de los negocios internacionales.

\section{Referencias}

Agmon, T. y Messica, A. (2009). Financial foreign direct investment: The role of private equity investments in the globalization of firms from emerging markets. Management International Review, 49(1), 11-26. doi: https://doi.org/10.1007/s11575-008-0122-9

Albort-Morant, G. y Ribeiro-Soriano, D. (2016). A bibliometric analysis of international impact of business incubators. Journal of Business Research, 69(5), 1775-1779. doi: https://doi.org/10.1016/j.jbusres.2015.10.054

Ankudinov, A., Ibragimov, R. y Oleg Lebedev. (2017). Sanctions and the Russian stock market. Research in International Business and Finance, 40, 150-162. doi: https://doi.org/10.1016/j.ribaf.2017.01.005

Ahmed, S. A. y Rock, J. (2012). Exploring the relationship between export intensity and exporter characteristics, resources, and capabilities: evidence from Chile. Latin American Business Review, 13(1), 29-57. doi: https:// doi.org/10.1080/10978526.2012.673423

Annan-Diab, F. y Filippaios, F. (2017). Multinational fir$\mathrm{ms}$ ' motivations and foreign direct investment decisions: an analysis of the software and IT and financial services sectors in the irish context. Thunderbird International Business Review, 59(6), 739-755.

Andriani, P. y Herrmann-Pillath, C. (2015). Transactional innovation as performative action: transforming comparative advantage in the global coffee business. Journal of Evolutionary Economics, 25(2), 371-400. doi: https://doi.org/10.1007/s00191-014-0388-y

Ancharaz, V. y Tandrayen-Ragoobur, V. (2013). Red dragon China and small Mauritius' trade links: an in-depth analysis. Journal of Chinese Economic and
Foreign Trade Studies, 6(3), 119-144. doi: https:/doi. org/10.1108/JCEFTS-10-2012-0018

Audretsch, B. (1998). Agglomeration and the location of innovative activity. Oxford review of economic policy, 14(2), 18-29. Recuperado de https://www.scopus.com/ inward/record.uri?eid=2-s2.0-33744970554\&partnerI$\mathrm{D}=40$ \&md5=bbbf0fd7f238107659dfa5f22577663e

Avkiran, N. K. (2017). An illustration of multiple-stakeholder perspective using a survey across Australia, China and Japan. Annals of Operations Research, 248(1-2), 93-121. doi: https://doi.org/10.1007/s10479016-2191-7

Bala Subrahmanya, M. H. (2007). Development strategies for Indian SMEs: promoting linkages with global transnational corporations. Management Research News, 30(10), 762-774. doi: https://doi. org/10.1108/01409170710823476

Banerjee, P. K., Chaudhari, M., Salunkhe, U. y Ravishankar, S. (2013). Opportunities and Control Measures for Sustainable Transport Growth in Emerging Economy Regions-India. SAE international, (2). doi: https://doi.org/10.4271/2013-01-1037

Bali, T.G., Brown, S.J. y Caglayan, M.O. (2016). Macroeconomic risk and hedge fund returns. Journal of Financial Economics, 114(1), 1-19. doi: https://doi. org/10.1016/j.jfineco.2014.06.008

Ben-Amor, R., Aguayo, E. y de Miguel-Gómez, M. D. (2015). The competitive advantage of the Tunisian palm date sector in the Mediterranean region. Spanish Journal of Agricultural Research, 13(2), 0101. doi: https://doi.org/10.5424/sjar/2015132-6390

Bond, E.W. (2013). Input quality, relational contracts and international outsourcing. Pacific Economic Review, 13(4), 391-404. doi: https://doi.org/10.1111/j.14680106.2008.00409.x

Brautigam, D., Weis, T. y Tang, X. (2018). Latent advantage, complex challenges: Industrial policy and Chinese linkages in Ethiopia's leather sector. China Economic Review, 48, 158-169. doi: https://doi.org/10.1016/j. chieco.2016.06.006

Cambra-Fierro, J. y Ruiz-Benítez, R. (2011). Sustainable business practices in Spain: a two-case study. European Business Review, 23(4), 401-412. doi: https://doi. org/10.1108/09555341111145780

Cary, J. y Roberts, A. (2011). The limitations of environmental management systems in Australian agriculture. Journal of Environmental Management, 92(3), 878-885. doi: https://doi.org/10.1016/j.jenvman.2010.10.055

Chang, D. R., Jang, J., Lee, E. Y., Lee, H. y Chang, B. (2017). When a good company transgresses: A study of the influences of CSR, moral decoupling, and ethnocentrism. 
Journal of Global Fashion Marketing, 8(1), 40-53. doi: https://doi.org/10.1080/20932685.2016.1255853

Contractor, F. J., Kumar, V. y Dhanaraj, C. (2015). Leveraging India: global interconnectedness and locational competitive advantage. Management International Review, 55(2), 159-179. doi: https://doi.org/10.1007/ s11575-015-0239-6

Cottier, T. (2015). The common law of international trade and the future of the World Trade Organization. Journal of International Economic Law, 18(1), 3-20. doi: https://doi.org/10.1093/jiel/jgv005

Dash, S. (2006, May). Human capital as a basis of comparative advantage equations in services outsourcing: A cross country comparative study. En International Conference on Information and Communication Technologies and Development. (pp. 165-175). Berkeley, Estados Unidos. doi: https://doi.org/10.1109/ ICTD.2006.301854

Demeter, K. y Szász, L. (2014). Exploring operations of manufacturing plant types in international context. International Operations Networks, 29-50. doi: https://doi. org/10.1007/978-1-4471-5646-8_3

Derado, D. (2011). Institutional conditions of small and medium enterprises' internationalization: The Case of the Split-Dalmatia county. Ekonomski Pregled, 62, 134-176. Recuperado de https://www.scopus.com/ record/display.uri?eid=2-s2.0-79955690520\&ori-

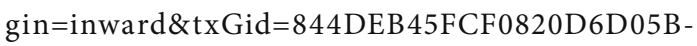
77F7AB087CF.wsnAw8kcdt7IPYLO0V48gA\%3a43

Enderwick, P. (2009). Applying the eclectic framework: The strategy of transnational criminal enterprises in the global era. Critical perspectives on international business, 5(3), 170-186. doi: https://doi. org/10.1108/17422040910974677

Eng, I. y Lin, Y. (1996). Seeking competitive advantage in an emergent open economy: foreign direct investment in Chinese industry. Environment and Planning A, 28(6), 1113-1138. Recuperado de https://www.scopus. com/inward/record.uri?eid=2-s2.0-0030432671\&partnerID $=40 \& \operatorname{md} 5=\operatorname{aad} 43 \mathrm{fd} 8 \mathrm{abadec} 8 \mathrm{a} 2191 \mathrm{f} 101 \mathrm{fb} 739 \mathrm{ad} 4$

Entrup, C. L. y Barth, T. (2008). On Problems, Requirements and Solution Approaches when Supporting Knowledge Intensive Processes in Industry. Knowledge Management In Action, 270, 111-124. Recuperado de https://www.scopus.com/inward/record.uri?eid=2-s2. $0-84902204827 \&$ partnerID $=40 \&$ md $5=1483 \mathrm{f} 0 \mathrm{~b}$ 4662532b84abe5d437f425586

Gan, L., Hernández, M. A. y Ma, S. (2016). The higher costs of doing business in China: Minimum wages and firms' export behavior. Journal of International Economics, 100, 81-94. doi: https://doi.org/10.1016/j. jinteco.2016.02.007
González, F., Flores, B., Gil-Lafuente, A.M., Flores, J. (2015). Uncertain Optimal Inventory as a Strategy for Enterprise Global Positioning. Decision Making and Knowledge Decision Support Systems, 675, 29-42. doi: https://doi.org/10.1007/978-3-319-03907-7_5

Harzing, A. W. y Giroud, A. (2014). The competitive advantage of nations: An application to academia. Journal of Informetrics, 8(1), 29-42. doi: https://doi.org/10.1016/j. joi.2013.10.007

Hung, C.-L. y Chou, J.C.-L (2014). Examining the cultural moderation on the acceptance of mobile commerce. International Journal of Innovation and Technology Management, 11(2), 1-19. doi: https://doi.org/10.1142/ S0219877014500102

Jensen, J. B. (2016). Overlooked Opportunity: Trade in Services. The Economists' Voice, 13(1), 1-7. doi: https:// doi.org/10.1515/ev-2016-0009

Javalgi, R. R. G., Gross, A. C., Benoy Joseph, W. y Granot, E. (2011). Assessing competitive advantage of emerging markets in knowledge intensive business services. Journal of Business \& Industrial Marketing, 26(3), 171-180. doi: https://doi.org/10.1108/08858621111115895

Kellow, A. (2012). Multi-level and multi-arena governance: the limits of integration and the possibilities of forum shopping. International Environmental Agreements: Politics, Law and Economics, 12(4), 327-342. doi: https://doi.org/10.1007/s10784-012-9172-3

Kilduff, P. y Chi, T. (2006). Longitudinal patterns of comparative advantage in the textile complex-Part 2: sectoral perspectives. Journal of Fashion Marketing and Management: An International Journal, 10(2), 150-168. doi: https://doi.org/10.1108/13612020610667478

Koch, W.L. (2007). A survey of China. En Electrical Insulation Conference and Electrical Manufacturing Expo (pp. 334-341). Nashvill, Estados Unidos. doi: https:// doi.org/10.1109/EEIC.2007.4562638

Kowalik, I., Danik, L. y Sikora, T. (2017). Entrepreneurial orientation elements in the Polish international new ventures. Baltic Journal of Management, 12(2), 194-213. doi: https://doi.org/10.1108/BJM-03-2016-0070

Krugman, P. R. (1992). Geografía y comercio. Barcelona, España: Antoni Bosch Editor.

Kundu, S. K. y Renko, M. (2005). Explaining export performance: a comparative study of international new ventures in Finnish and Indian software industry. En J. Katz y A. Corbett (Eds.), Advances in entrepreneurship, firm emergence and growth (pp. 43-84). Londres, Inglaterra: Emerald Publishing Limited. doi: https:// doi.org/10.1016/S1074-7540(05)08003-7 
Kuznetsov, A.V. (2016). Transnational corporation studies in Imemo: Achievements and new tasks. World Economy and International Relations, 60(12), 111-117. doi: https://doi.org/10.20542/0131-2227-2016-60-12-111-117

Kulubekova, G., Viglione, F. y Busurmanov, Z. (2016). Enhancement of the regional business legal structure within the Eurasian economic union based on the use of the European Union's experience. Indian Journal of Science and Technology, 9(21), 1-8. doi: 10.17485/ ijst/2016/v9i21/95190

Kysel'ová, V. (2014). Industry as a key factor for economic recovery and competitiveness of the Europe. Applied Mechanics and Materials, 613, 441-445. doi: https:// doi.org/10.4028/www.scientific.net/AMM.613.441

Laguna, N. M. (2004). Oil policies and privatization strategies in Mexico: implications for the petrochemical sector and its production spaces. Energy Policy, 32(18), 2035-2047. doi: https://doi.org/10.1016/S03014215(03)00179-4

Iammarino, S., McCann, P. y Ortega-Argilés, R. (2018). International business, cities and competitiveness: recent trends and future challenges. Competitiveness Review: An International Business Journal, 28(3), 236-251. doi: https://doi.org/10.1108/CR-10-2017-0070

Lee, S. M. y Rha, J. S. (2018). A network text analysis of published papers in service business, 2007-2017: research trends in the service sector. Service Business, 12(4), 809-831. doi: https://doi.org/10.1007/s11628018-0377-6

Lemoine, F. (2013). From foreign trade to international investment: a new step in China's integration with the world economy. Economic Change and Restructuring, 46(1), 25-43. doi: https://doi.org/10.1007/s10644-0129134-3

Li, N., Toppinen, A. y Lantta, M. (2016). Managerial Perceptions of SMEs in the Wood Industry Supply Chain on Corporate Responsibility and Competitive Advantage: Evidence from China and Finland. Journal of Small Business Management, 54(1), 162-186. doi: https://doi.org/10.1111/jsbm.12136

Lin, J.-B. y Fu, S.-H. (2016). Investigating the dynamic relationships between equity markets and currency markets. Journal of Business Research, 69(6), 2193-2198. doi: https://doi.org/10.1016/j.jbusres.2015.12.029

Luiz, J., Stringfellow, D. y Jefthas, A. (2017). Institutional complementarity and substitution as an internationalization strategy: the emergence of an African multinational giant. Global Strategy Journal, 7(1), 83-103. doi: https://doi.org/10.1002/gsj.1143
Luo, Y. y Zhang, H. (2016). Emerging market MNEs: Qualitative review and theoretical directions. Journal of International Management, 22(4), 333-350. doi: https:// doi.org/10.1016/j.intman.2016.05.001

Malik, M. H. y Velan, N. (2017). Trends and determinants of IT-BPM exports in India. Journal of Science and Technology Policy Management, 7(2), 212-232. doi: https://doi.org/10.1108/JSTPM-08-2015-0028

Malik, T. (2012). First mover, strategic alliances and performance: context of turmoil in China. Chinese Management Studies, 6(4), 647-667. doi: https://doi. org/10.1108/17506141211280308

McNamara, D. L. (2006). New places but old spaces: knowledge hierarchies among Asian small and medium size enterprises abroad. Organization, 13(4), 549567. doi: https://doi.org/10.1177/1350508406065105

Martek, I. y Chen, C. (2016). Value chain supply procurement strategies in international construction: cases of foreign constructors in China. Management Decision, 54(2), 501-521. doi: https://doi.org/10.1002/tie.21743

Montenegro, Y. A. y Valbuena, P. N. (2018). Tendencias de investigación en relacionamiento legal en los negocios internacionales (2012-2016). Espacios, 39(03), 1-14 Recuperado de http://www.revistaespacios.com/ a18v39n03/a18v39n03p02.pdf

Moroni, I., Arruda, A., Bezerra, P. y Laila, T. (2018). How the design processes add innovative capacity in startup companies. Advances in Intelligent Systems and Computing, 588, 469-476. doi: https://doi.org/10.1007/9783-319-60582-1_47

Myers, M. B., Fawcett, S. E.y Smith, S. R. (2002). International production sharing opportunities in Mexico and the Caribbean: A comparative study of manufacturing and logistics efforts. Latin American Business Review, 3(2), 65-84. doi: https://doi.org/10.1300/ J140v03n02_04

Naray, O. y Bezençon, V. (2017). Management and business research on commercial diplomacy: Examining trends and themes. The International Trade Journal, 31(4), 332-359.

Nguyen, T. T. y Nguyen, T. D. (2010). Learning to build quality business relationships in export markets: Evidence from Vietnamese exporters. Asia Pacific business review, 16(1-2), 203-220. doi: https:/doi. org/10.1080/13602380802280009

Norberg-Hodge, H. (2006). Sustainable Economies-Local or Global? En M. Keiner (Ed.). The future of sustainability (pp. 99-115). Dordrecht: Springer. doi: https://doi. org/10.1007/1-4020-4908-0_5

Palekhova, L. (2016). Achieving the competitiveness through public accountability on sustainable development. 
Scientific Bulletin of National Mining University, (2), 162-167. Recuperado de https://www.scopus.com/ record/display.uri?eid=2-s2.0-84978132460\&origin=inward\&txGid=844DEB45FCF0820D6D05B77F7AB087CF.wsnAw8kcdt7IPYLO0V48gA\%3a26

Patel, P. y Pavitt, K. (1994). National innovation systems: why they are important, and how they might be measured and compared. Economics of innovation and new technology, 3(1), 77-95. doi: https://doi. org/10.1080/10438599400000004

Parausic, V., Potrebic, V. y Simonovic, Z. (2013). Factor Advantages of the Republic of Serbia in Agricultural Production and a Need for Accomplishment of Sustainable Competitive Advantage in the Market. En A. Jean-Vasile, T. Adrian, J. Subic y D. Dusmanescu (Eds.), Sustainable Technologies, Policies, and Constraints in the Green Economy (pp. 63-79). Hershey, Estados Unidos: IGI Global. doi: https://doi.org/10.4018/978-14666-4098-6.ch004

Pearce, J. L., Xin, K. M., Xu, Q. J. y Rao, A. N. (2011). Managers' context: how government capability affects managers. British Journal of Management, 22(3), 500-516. doi: https://doi.org/10.1111/j.1467-8551.2011.00756.x

Rajagopal, R (2009). Globalization Thrust: Driving Nations Competitive. New York : Nova Science Publishers.

Rajapriya, M., Kumar, N. y Arul Krishnan, S. (2017). An empirical study on work related stress faced by the employees across the business processing outsourcing (BPO'S) in Chennai. International Journal of Economic Research, 14(1), 23-36. Recuperado de https://www.scopus.com/record/ display.uri?eid=2-s2.0-85016477190\&origin=inward\&txGid=143A7CA80234B124F14897348292CA0B.wsnAw8kcdt7IPYLO0V48gA\%3a1

Renchu, Y. J. G. (1999). Comparative Analysis of Business Management Between Chinese and Japanese Manufacturing Enterprieses. Journal of Beijing Institute of Technology, 19(4), 407-411. Recuperado de https://www.scopus.com/inward/record.uri?ei$\mathrm{d}=2$-s2 .0 -71149104037\&partnerID $=40 \& \mathrm{md} 5=\mathrm{f}-$ 10b00aec56af854d907d12f6b6f2cdd

Regnér, P. y Edman, J. (2014). MNE institutional advantage: How subunits shape, transpose and evade host country institutions. Journal of International Business Studies, 45(3), 275-302. doi: 10.1057/jibs.2013.66

Rozkwitalska, M., Chmielecki, M., Przytula, S., Sulkowski, L. y Basinska, B.A. (2017). Intercultural interactions in multinational subsidiaries: Employee accounts of "the dark side" and "the bright side" of intercultural contacts. Baltic Journal of Management, 12(2), 214-239. doi: https://doi.org/10.1108/BJM-11-2015-0215
Ruamsook, K., Russell, D. M. y Thomchick, E. A. (2009). Sourcing from low-cost countries: Identifying sourcing issues and prioritizing impacts on logistics performance. The International Journal of Logistics Management, 20(1), 79-96. doi: https://doi. org/10.1108/09574090910954855

Saraswat, S. P. (2012). Meeting the Challenge of Globalization with Information and Communications Technology at an Emerging Multinational Enterprise. En 18th Americas Conference on Information Systems 2012 (pp. 4407-4415). Recuperado de https://www.scopus.com/ inward/record.uri?eid=2-s2.0-84878084693\&partne$\mathrm{rID}=40 \& \mathrm{md} 5=\mathrm{f} 348 \mathrm{cf} 2 \mathrm{f} 6 \mathrm{fc} 6 \mathrm{a} 3732 \mathrm{ec} 60680915 \mathrm{~d} 16 \mathrm{ce}$

Selig, G. J. (1982). A framework for multinational information systems planning. Information \& Management, 5(2), 95-115. doi: 10.1016/0378-7206(82)90042-8

Sharif, M. N. (1997). Technology strategy in developing countries: evolving from comparative to competitive advantage. International Journal of $\mathrm{Te}$ chnology Management, 14(2-4), 309-343. Recuperado de https:/www.scopus.com/inward/record.uri?eid=2s2.0-0030713155\&partnerID $=40 \& \mathrm{md} 5=5 \mathrm{fc} 73285 \mathrm{a} 6 \mathrm{a}-$ 653972957bf3bef7d90f6

Skorupinska, A. (2017). ICT, Innovation and Productivity: Evidence Based on Eastern European Manufacturing Companies. Journal of the Knowledge Economy, 8(2), 768-788. doi: https://doi.org/10.1007/s13132-016-0441-1

Silvia, J. (2006). Domestic implications of a global labor market. Business Economics, 41(3), 23-29. doi: https:// doi.org/10.2145/20060302

Taneja, N., Mehra, M., Mukherjee, P., Bimal, S. y Dayal, I. (2015). Normalizing India-Pakistan Trade. En N. Taneja y S. Pohit (Eds.), India-Pakistan Trade (pp. 13-45). New Delhi, India: Springer. doi: https:// doi.org/10.1007/978-81-322-1949-1_2

Templin, P. (2010). The free trade myth. Industrial Engineer, 42(5), 26-27. Recuperado de https:/www.scopus.com/ inward/record.uri?eid=2-s2.0-77952772992\&partne$\mathrm{rID}=40 \& \mathrm{md} 5=774331 \mathrm{f} 32 \mathrm{~d} 863 \mathrm{efb} 1 \mathrm{e} 1 \mathrm{e} 9 \mathrm{~d} 5 \mathrm{~b} 7 \mathrm{ac} 732 \mathrm{dc}$

Thompson, E. R. (2001). Dangers of differential comprehensions of Hong Kong's competitive advantages: Evidence from firms and public servants. The China Quarterly, 167, 706-723. Recuperado de https://www.scopus.com/ inward/record.uri?eid=2-s2.0-0035171917\&partnerI$\mathrm{D}=40 \& \mathrm{md} 5=\mathrm{d} 18 \mathrm{e} 1 \mathrm{f} 0 \mathrm{eb} 68 \mathrm{c} 2 \mathrm{~d} 746302740 \mathrm{a} 23 \mathrm{a} 26 \mathrm{e} 5 \mathrm{~d}$

Torok, A. y Jambor, A. (2016): Determinants of the revealed comparative advantages: The case of the European ham trade. CAAS Agricultural Journals, 62, 471-482. doi: https://doi.org/10.17221/177/2015-AGRICECON

Toulmin, S. (1999). The ambiguities of globalization. Futures, 31(9), 905-912. doi: https://doi.org/10.1016/S00163287(99)00049-X 
Tsang, D. (2006). The entrepreneurial culture: network advantage within Chinese and Irish software firms. Cheltenham, Inglaterra: Edward Elgar Publishing. Recuperado de https://www.scopus.com/inward/record. uri?eid=2-s2.0-84866012440\&partnerID =40\&md5=53198caca9f69e1c119be59efb67d16d

Ushakov, D. y Arkhipov, A. (2013). Urban business systems' globalization: Factors, forms and economic impact. Research Journal of Business Management, 8(3), 189202. doi: https://doi.org/10.3923/rjbm.2014.189.202

Valbuena H., P. N. y Montenegro J., Y. (2017). Tendencias de investigación en negocios internacionales (2012-2016). Cuadernos Latinoamericanos de Administración, 13(25), 9-18. doi: http://dx.doi.org/10.18270/cuaderlam.v13i25.2385

Vrana, V. y Zafiropoulos, K. (2009). Rural tourism lodgings' websites: a comparative study among Mediterranean countries. International Journal of Tourism Policy, 2(1-2), 89-106. doi: https://doi.org/10.1504/ IJTP.2009.023275

Wang, C., Li, X. y Mao, Z. (2013). Green Retailing Practices: An Exploratory Comparison between Chinese and British Retailers. Journal of General Management, 39(2), 35-60. doi: https://doi. org/10.1177/030630701303900203

Walter, A. y Segerstedt, A. (2012). International trade of biofuels: current trends and the potential role of Africa. En R. Janssen y D. Rutz (Eds.), Bioenergy for sustainable development in Africa (pp. 147-162). Dordrecht, Holanda: Springer Netherlands. doi: https:// doi.org/10.1007/978-94-007-2181-4-13

Wei, S. y Joyce, D. (2016, agosto). Floricultural value chain case studies in Fiji, Solomon Islands and Papua New Guinea. En XXIX International Horticultural Congress on Horticulture: Sustaining Lives, Livelihoods and Landscapes. Brisbane, Australia. doi: https://doi. org/10.17660/ActaHortic.2016.1129.7

White, G. O., Guldiken, O., Hemphill, T. A., He, W. y Khoobdeh, M. S. (2016). Trends in international strategic management research from 2000 to 2013: text mining and bibliometric analyses. Management International Review, 56(1), 35-65. doi: https://doi. org/10.1007/s11575-015-0260-9

Williamson, P. J. (2014). The global expansion of EMNCs: paradoxes and directions for future research. Cuervo, A. y Ramamurti, R., Understanding Multinationals from Emerging Markets (pp. 155-168). Cambridge University Press. doi: https://doi.org/10.1017/ CBO9781107587632.012

Winters, L. A., Walmsley, T. L., Wang, Z. K. y Grynberg, R. (2003). Liberalising temporary movement of natural persons: an agenda for the development round. The World Economy, 26(8), 1137-1161. Recuperado de https:/www.scopus.com/inward/record. uri?eid=2-s2.0-0141683493\&partnerID $=40 \& \mathrm{md} 5=-$ $5560480916 \mathrm{~b} 7336139515 \mathrm{~b} 8632 \mathrm{cfda} 80$

Wilson, J. y Brennan, R. (2003). Market entry methods for Western firms in China. Asia Pacific Journal of Marketing and Logistics, 15(4), 3-18. doi: https://doi. org/10.1108/13555850310765015

Williams, T., Al-Mejlad, F., Al-Naimi, F., Freens, P.,Taha, B., Sarkova, V., ..., Sharifi, A.R. (2015). Life cycle assessment of LNG. En International Gas Union World Gas Conference Papers. París, Francia. Recuperado de https://www.scopus.com/inward/record. uri?eid=2-s2.0-84963612288\&partnerID $=40 \& \operatorname{md} 5=-$ d562e76ec1b8b0cf3b4d9c8e5c14cba3

Wysokińska, Z. y Witkowska, J. (2005). International Business and Environmental Issues-Some Empirical Evidence from Transition Economies. Polish Journal of EnvironmentalStudies, 14(3),269-279. Recuperadodehttps://www.scopus.com/inward/record.uri?eid=2-s2.0$18544362643 \&$ partnerID $=40 \&$ md $5=$ a 29d $230165 f-$ 6168baf79d782e45f 1418

Yamori, N. (1997). Do Japanese banks lead or follow international business? An empirical investigation. Journal of International Financial Markets, Institutions and Money, 7(4), 369-382. Recuperado de https://www.scopus. com/inward/record.uri?eid=2-s2.0-0031536924\&partnerID $=40 \& m d 5=b f 82 f 0638 f 45 a 70 f e 458 c c 893 e a c 2 d 19$

Yang, D., Sonmez, M. M., Li, Q. y Duan, Y. (2015). The power of triple contexts on customer-based brand performance- - A comparative study of Baidu and Google from Chinese netizens' perspective. International Business Review, 24(1), 11-22. doi: https://doi. org/10.1016/j.ibusrev.2014.05.004

Yeoh, P. L. (2011). Location choice and the internationalization sequence: Insights from Indian pharmaceutical companies. International Marketing Review, 28(3), 291-312. doi: https://doi.org/10.1108/02651331111132866

Zostautiene, D., Susniene, D., Purvinis, O., Sargunas, G. y Puckyte, I. (2016). Disclosure of social responsibility practices at multinational enterprises: Empirical evidence for Lithuania. Milán, Italia: International Business Information Management Association. Recuperado de https://www.scopus.com/record/ display.uri?eid=2-s2.0-84984624661\&origin=inwar$\mathrm{d} \& \mathrm{txGid}=143 \mathrm{~A} 7 \mathrm{CA} 80234 \mathrm{~B} 124 \mathrm{~F} 14897348292 \mathrm{CA0B}$. wsnAw8kcdt7IPYLO0V48gA\%3a9 